\title{
Nuclear Medicine in Cancer Theranostics: Beyond the Target
}

\author{
David Taïeb ${ }^{1}$, Rodney J. Hicks², and Karel Pacak ${ }^{3}$ \\ ${ }^{1}$ Department of Nuclear Medicine, La Timone University Hospital, CERIMED, Aix-Marseille University, Marseille, France; ${ }^{2}$ Cancer \\ Imaging and Neuroendocrine Tumour Service, Sir Peter MacCallum Department of Oncology, Peter MacCallum Cancer Centre, \\ University of Melbourne, Melbourne, Australia; and ${ }^{3}$ Section on Medical Neuroendocrinology, Eunice Kennedy Shriver National \\ Institute of Child Health and Human Development, National Institutes of Health, Bethesda, Maryland
}

O ncologic nuclear medicine has come to a fork in the road, with the choice being whether to remain a peripheral diagnostic lesion-detection technique competing against the ever-evolving anatomic imaging modalities or whether to join the revolution in precision medicine, characterizing tumor biology and directing treatment through highly specific tracers. The term theranostics encapsulates the integration of diagnostics and therapeutics in the individualized management of disease (1). Implicit in the theranostic paradigm is the assumption that diagnostic test results can precisely determine whether an individual is likely to benefit from a specific treatment. This assumption underpins the recent focus on companion diagnostics as an integral part of drug development. An excellent example of the concept of theranostics in oncology is the requirement that, to be selected for trastuzumab therapy (Herceptin; Genentech), a candidate must have a tumor on which the presence of human epidermal growth factor receptor 2 (HER2) has been demonstrated. However, this requirement is limited by the potential sampling bias intrinsic in tissue biopsy. Molecular imaging of HER2 expression using ${ }^{89} \mathrm{Zr}$-radiolabeled trastuzumab provides an alternative vision of how the selection of candidates for expensive and sometimes toxic therapies might look in the future (2). Molecular imaging with ${ }^{89} \mathrm{Zr}$-trastuzumab can be used to detect heterogeneity of HER2 expression. Because it can image the whole body, it has the potential to improve selection of patients for trastuzumab and antibody-drug conjugate therapy (3). It also opens the way for therapeutic application of radionuclide therapy (4). Beyond the expected clinical benefits of personalized medicine, theranostics could also have a significant positive economic effect. For example, KRAS is an oncoprotein that acts downstream of the epidermal growth factor receptor and thereby predicts a poor response to anti-epidermal growth factor receptor therapies. The implementation of KRAS mutation status as a companion test to exclude patients unlikely to benefit from such therapies has been estimated to save a net cost of $\$ 7,500-\$ 12,400$ per patient in the United States and $€ 3,900-€ 9,600$ per patient in Germany, with equivalent clinical outcomes (5). This economic impact becomes particularly relevant when one is contemplating introducing relatively expensive imagingbiomarker studies. Given the huge relative costs of modern cancer

\footnotetext{
Received May 16, 2016; revision accepted May 20, 2016.

For correspondence or reprints contact: David Taïeb, Department of Nuclear Medicine, La Timone University Hospital, European Center for Research in Medical Imaging, Aix-Marseille University, 264 rue Saint-Pierre, 13385 Marseille, France.

E-mail: david.taieb@ap-hm.fr.

Published online Jul. 21, 2016

COPYRIGHT (C) 2016 by the Society of Nuclear Medicine and Molecular Imaging, Inc.

DOI: $10.2967 /$ jnumed.116.178343
}

therapies, the costs of imaging could be more than adequately offset by preventing futile or unnecessary treatments.

Nuclear medicine is ideally placed to play a central role in theranostics by allowing visualization of molecular targets and thus enabling so-called in vivo immunohistochemistry, by which noninvasive biomarkers can be provided to select targeted drugs labeled with therapeutic radionuclides and monitor the response to them. The staging and treatment of thyroid cancer-with the diagnostic use of ${ }^{123} \mathrm{I}$ or ${ }^{124} \mathrm{I}$ complementing the therapeutic efficacy of ${ }^{131} \mathrm{I}$ - has paved the way for theranostics in therapeutic nuclear medicine. Successful treatment of metastatic thyroid cancer was achieved even before the molecular basis of radioiodine uptake through the sodium-iodide symporter was characterized, speaking to the power of this paradigm. The use of radiolabeled metaiodobenzylguanidine in diagnosis and treatment of metastatic neuroblastoma, paraganglioma, and pheochromocytoma or of radiolabeled somatostatin analogs in neuroendocrine tumors (NETs) has extended the paradigm to other cancers. Despite the impressive results achieved using these agents, they have generally been developed in academic centers and used on a compassionate basis. This has led to limited resources for establishing the evidence base that usually accompanies registration and approval of cancer therapies. In particular, there has been a lack of randomized controlled trial data comparing radionuclide therapies with other forms of therapy and virtually none testing the integrated theranostic approach. This failing in the eyes of the oncology community has recently been addressed in the case of small intestinal NET, after the recent presentation of the results of the NETTER-1 study at the 2016 Gastrointestinal Cancers Symposium. Briefly, the NETTER-1 study is a randomized controlled trial that is comparing ${ }^{177} \mathrm{Lu}$-DOTATATE (4 administrations of $7.4 \mathrm{GBq}$ ) with an augmented dose of long-actingrelease octreotide $(60 \mathrm{mg})$ in patients with progressive, somatostatinreceptor-expressing midgut NETs (ileal in 75\% of cases). The interim analysis is encouraging for ${ }^{177} \mathrm{Lu}$-DOTATATE, with an objective response of $19 \%$ (vs. 3\%) and an increase in progressionfree survival (not reached but estimated to be 40 mo for peptide receptor radionuclide therapy [PRRT] vs. 8.4 mo with augmented-dose long-acting-release octreotide). Although there is as yet no randomized controlled trial evidence supporting the utility of an increased dose of somatostatin analogs as an antiproliferative therapy, it is interesting to note that most previously progressing patients in this arm of the trial achieved disease stabilization on this therapy, supplementing data from the PROMID (6) and CLARINET (7) studies that suggest that these agents can themselves delay disease progression. This makes the results even more impressive than those achieved using targeted agents, which have generally been tested against a placebo. Although the 
overall survival data are not yet mature and median survival has not been reached in either arm, there was reduced mortality in the PRRT arm (13 vs. 22 cases). What is important for an essentially palliative therapy is that the toxicity profile of PRRT was acceptable. We believe that these data will provide a powerful impetus for wider application of the ${ }^{68} \mathrm{Ga} /{ }^{177} \mathrm{Lu}$-DOTATATE strategy in the management of inoperable grade 1-2 intestinal NETs that progress despite use of somatostatin analogs. Clinical trials comparing ${ }^{177}$ Lu-DOTATATE to small-molecule inhibitors in pancreatic NETs are currently ongoing. Likewise, somatostatin receptor antagonists have also provided promising results for somatostatin receptor targeting (8), but these somatostatin receptor antagonists will likely require head-to-head comparison with other treatments of NET to assess their relative efficacy and toxicity. These encouraging results have expanded the scope of theranostics to other targets, such as ${ }^{68} \mathrm{Ga} /{ }^{177} \mathrm{Lu}$-labeled prostate-specific membrane antigen ligands in prostate cancer (9) or ${ }^{68} \mathrm{Ga} /{ }^{177} \mathrm{Lu}$-labeled chemokine receptor 4 ligands in myeloproliferative syndromes and solid tumors (10).

If the theranostic paradigm is to become established as a valid method of treating cancers, we believe it is important to learn from both the successes and the failures of the past. The evolution of thyroid cancer management provides an illustration of the key principles required to advance a theranostic approach. These include...

- Optimal patient selection. This principle must be based on a knowledge of not only target expression but also other prognostic factors that influence outcomes, such as tumor burden, tumor location, and the presence and biologic behavior of subclones of cells lacking target expression. For radioiodine therapy, these factors include the presence of non-iodine-avid lesions, particularly those with intense ${ }^{18}$ F-FDG uptake, a short doubling time for thyroglobulin, large tumor volumes, the presence of bone metastases, and significant local invasion. For PRRT, the corresponding factors that need to be considered are spatial discordance between somatostatin receptor avidity and ${ }^{18}$ F-FDG avidity, disease burden, proliferative activity, and possibly the site of the primary tumor.

- Optimization of the therapeutic index by prospective dosimetry. This principle, although not yet widely applied or clearly validated, is desirable. Wider availability of ${ }^{124}$ I potentially enables the therapeutic index to be optimized for ${ }^{131}$ I therapy and quantitative SPECT/CT, or longer-lived somatostatin receptor ligands such as ${ }^{64} \mathrm{Cu}$-labeled somatostatin analogs might make this optimization feasible for PRRT.

- The ability to pharmacologically manipulate target expression. For radioiodine therapy, pharmacologic manipulation of target expression involves upregulation of iodine transporter expression through increased thyrotropin levels (endogenous or exogenous) and, more recently, through use of tyrosine kinase inhibitors in the mitogen-activated protein kinase pathway in iodine-refractory thyroid cancer (11). For PRRT, somatostatin analog therapy may influence somatostatin receptor expression.

- Use of cytostatic treatments between and after radionuclide therapy. An example of this principle is the use of supraphysiologic thyroid hormone replacement to achieve thyrotropin suppression after radioactive iodine. Data from the PROMID and CLARINET studies cited above indicate that for PRRT, ongoing use of somatostatin analogs may delay recrudescent disease.

- Optimal patient follow-up using accurate, reliable markers of therapeutic efficacy. For ${ }^{131} \mathrm{I}$, these markers include a decreasing level of serum thyroglobulin and a decrease in (or preferably absence of) radioiodine uptake sites on posttherapy scintigraphy with a concomitant absence of morphologic progression or resolution of targeted lesions. Similarly, for PRRT, evidence of a response can be provided by chromogranin A or any specific bioamine or hormone produced by functioning NET, combined with repeated somatostatin receptor imaging and anatomic imaging.

Therefore, despite being somewhat specific, the radioiodine theranostic model should serve as a template for improving the efficacy of and evidence base for therapeutic nuclear medicine. Although immediately applicable to PRRT, the template is also relevant for a range of other radionuclide therapies. There is particular excitement, for example, about targeting the prostatespecific membrane antigen in prostate cancer, but we need to ensure that this application is developed in a rigorous, scientific environment that defines the best therapeutic windows, optimizes tumor targeting and radiopharmaceutical delivery, minimizes exposure of at-risk organs to radiation (e.g., dosimetry-based, mathematic modeling), selects the most suitable isotopes according to the targeted tissue compartment (Auger electrons, $\alpha$ - and $\beta$-emitters), identifies agents that can modulate target expression or increase radiation-induced cellular damage (radiosensitizing agents), and encourages the combination of cytostatic treatments between radioactive sessions while identifying reliable and accurate biomarkers of therapeutic response. To achieve all this, an active collaboration between different implied disciplines is necessary.

\section{DISCLOSURE}

No potential conflict of interest relevant to this article was reported.

\section{REFERENCES}

1. Baum RP, Kulkarni HR. THERANOSTICS: from molecular imaging using Ga68 labeled tracers and PET/CT to personalized radionuclide therapy-the Bad Berka experience. Theranostics. 2012;2:437-447.

2. Dijkers EC, Kosterink JG, Rademaker AP, et al. Development and characterization of clinical-grade ${ }^{89} \mathrm{Zr}$-trastuzumab for HER2/neu immunoPET imaging. $J$ Nucl Med. 2009;50:974-981.

3. Gebhart G, Lamberts LE, Wimana Z, et al. Molecular imaging as a tool to investigate heterogeneity of advanced HER2-positive breast cancer and to predict patient outcome under trastuzumab emtansine (T-DM1): the ZEPHIR trial. Ann Oncol. 2016;27:619-624.

4. Persson M, Tolmachev V, Andersson K, Gedda L, Sandstrom M, Carlsson J. $\left[{ }^{177} \mathrm{Lu}\right]$ pertuzumab: experimental studies on targeting of HER-2 positive tumour cells. Eur J Nucl Med Mol Imaging. 2005;32:1457-1462.

5. Vijayaraghavan A, Efrusy MB, Goke B, Kirchner T, Santas CC, Goldberg RM. Cost-effectiveness of KRAS testing in metastatic colorectal cancer patients in the United States and Germany. Int J Cancer. 2012;131:438-445.

6. Rinke A, Muller HH, Schade-Brittinger C, et al. Placebo-controlled, double-blind, prospective, randomized study on the effect of octreotide LAR in the control of tumor growth in patients with metastatic neuroendocrine midgut tumors: a report from the PROMID study group. J Clin Oncol. 2009;27:4656-4663.

7. Caplin ME, Pavel M, Cwikla JB, et al. Lanreotide in metastatic enteropancreatic neuroendocrine tumors. N Engl J Med. 2014;371:224-233.

8. Dalm SU, Nonnekens J, Doeswijk GN, et al. Comparison of the therapeutic response to treatment with a ${ }^{177} \mathrm{Lu}$-labeled somatostatin receptor agonist and antagonist in preclinical models. J Nucl Med. 2016;57:260-265.

9. Baum RP, Kulkarni HR, Schuchardt C, et al. ${ }^{177} \mathrm{Lu}$-labeled prostate-specific membrane antigen radioligand therapy of metastatic castration-resistant prostate cancer: safety and efficacy. J Nucl Med. 2016;57:1006-1013.

10. Herrmann K, Schottelius M, Lapa C, et al. First-in-human experience of CXCR4-directed endoradiotherapy with ${ }^{177} \mathrm{Lu}$ - and ${ }^{90}$ Y-labeled pentixather in advanced-stage multiple myeloma with extensive intra- and extramedullary disease. J Nucl Med. 2016;57:248-251.

11. Pattison DA, Solomon B, Hicks RJ. A new theranostic paradigm for advanced thyroid cancer. $J$ Nucl Med. March 31, 2016 [Epub ahead of print]. 